\title{
Virtual Stick Balancing: Statistical Invariants of Human Response
}

\author{
Shigeru Kanemoto, Ihor Lubashevsky*, Arkady Zgonnikov, \\ Toru Miyazawa, Daichi Taniguchi \\ University of Aizu, Tsuruga, Ikki-machi, \\ Aizu-Wakamatsu City, Fukushima 965-8560, Japan \\ *E-mail: i-lubash@u-aizu.ac.jp
}

\begin{abstract}
Human behavior during the process of virtual inverted pendulum balancing in viscous environment is analyzed. The results of the virtual experiments are compared to the results of previous studies on so called dynamical trap effect. It is shown that the phase trajectories and phase variables distributions of the virtual stick motion under human control are similar to those of an oscillator under the presence of noise described by the dynamical trap model. Moreover, it is discovered that the patterns of system dynamics under human control are similar for all feasible values of system parameters. We therefore suggest that the dynamical trap model could reflect certain features of human behavior during processes of dynamical systems control near equlibrium points.
\end{abstract}

\section{Introduction}

During the last decades there has been a notable progress in describing social systems and human behavior based on physical notions and mathematical formalism developed in statistical physics and applied mathematics (see, e.g., [1]). One of the specialized notions used for modeling of some essential properties of human cognition is the concept of dynamical traps [2].

Let us consider a hypothetical dynamical system controlled by a human operator whose purpose is to stabilize the system near an equilibrium point. We assume that the operator does not react to small deviations from the equilibrium, though these variations could be recognized by her perception. In other words, the operator is comfortable with the deviations of a small magnitude. Therefore, any point from a certain neighborhood of the equilibrium one is treated equally by the operator. This phenomenon is due to the effect of human fuzzy rationality [3]. The notion of dynamical traps was previously introduced in order to mimic this feature of the bounded capacity of human cognition [2].

\section{Dynamical traps}

In order to illustrate the dynamical traps concept, let us assume that considered hypothetical dynamical system is described by the following equations

$$
\begin{aligned}
& \dot{x}=y, \\
& \dot{y}=\Omega(x, y) F(x, y)+\xi(t),
\end{aligned}
$$

and has an equilibrium in the origin of coordinates $(0,0)$. Here $\Omega(x, y)$ stands for the dynamical trap effect, $F(x, y)$ is the sum of the regular forces (implicitly including human control) and $\xi(t)$ is the random factor. $\Omega(x, y)$ could be defined as follows

$$
\begin{aligned}
& \Omega(x, y) \approx 0 \text { if }(x, y) \in \mathbb{Q}_{\mathrm{tr}}, \\
& \Omega(x, y)=1 \quad \text { otherwise }
\end{aligned}
$$

where $\mathbb{Q}_{\text {tr }}$ is a certain vicinity of the equilibrium point.

In order to explain the meaning of cofactor $\Omega(x, y)$ we consider the behavior of the operator who is approaching the desired phase space position $(x=0, y=0)$. Let us assume that if the current position is far from the origin, the operator perfectly follows the optimal control strategy. If the current position is recognized by the operator as "good enough" $\left((x, y) \in \mathbb{Q}_{t r}\right.$, i.e. it may not be strictly optimal) due to her fuzzy rationality, she halts active control over the system so that system dynamics is stagnated in certain vicinity of the desired position. Therefore, $\mathbb{Q}_{t r}$ is called the region of dynamical traps.

The investigation of the dynamical traps model was originally inspired by a class of intrinsic cooperative phenomena found in the dynamics of vehicle ensembles on highways [4]; later it was shown that the dynamical trap effect could cause emergent phenomena in the chain of oscillators mimicking the interaction of motivated objects $[2,5]$. Among other results obtained in [5], it was demonstrated numerically that the "motivated" oscillator from the particle chain under the presence of dynamical trap forms the specific phase space trajectories (see Fig. 1). The phase variables distributions were shown to take non-Gaussian forms. The reviewed results demonstrate that the dynamical 

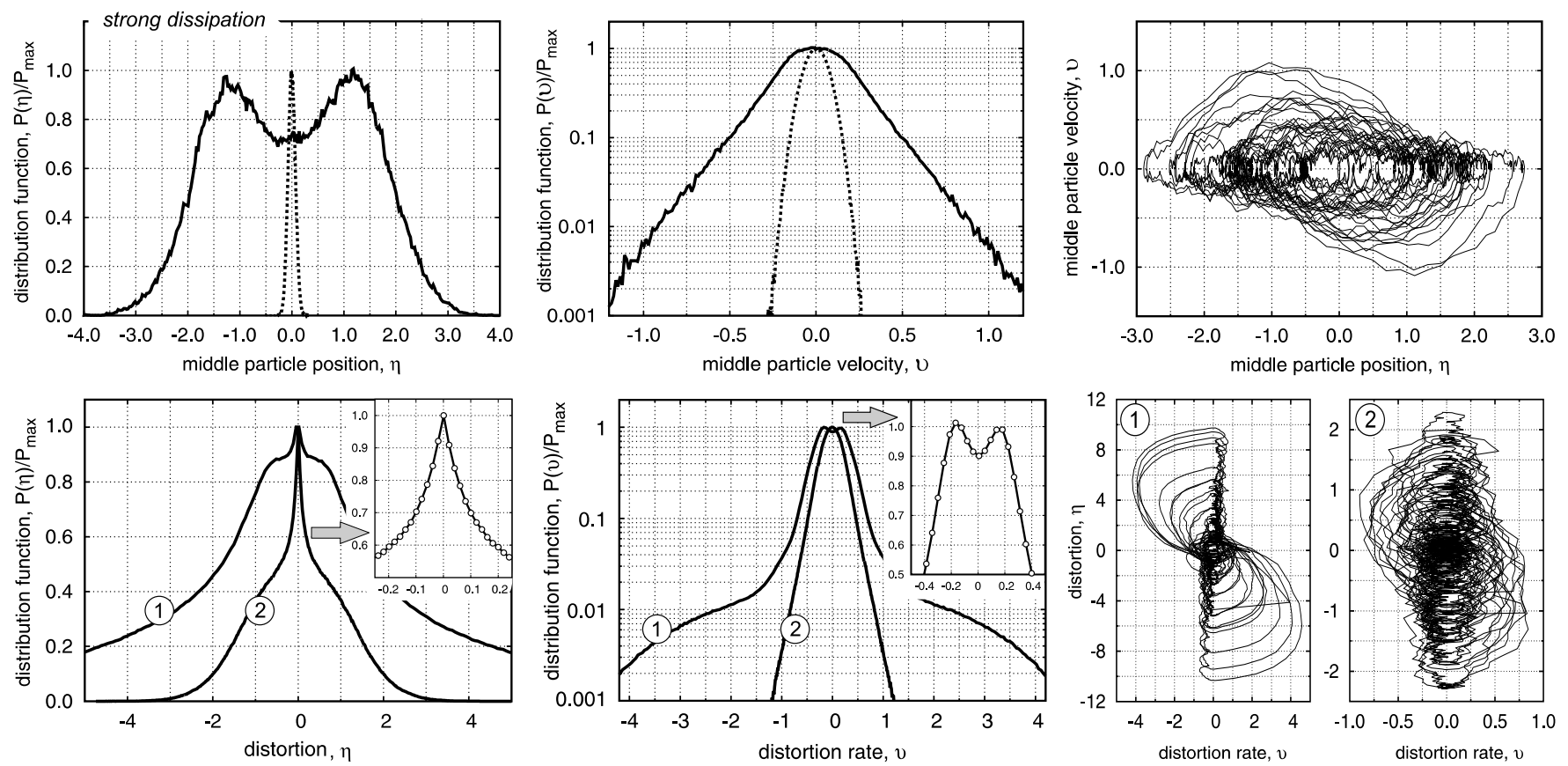

Fig. 1: Phase variables density functions and phase trajectories of a single particle from the ensemble of interacting motivated particles governed by fuzzy rationality [5]. The top three diagrams correspond to the case of the single moving particle oscillating between two fixed neighbors. On the density diagrams solid lines correspond to the case of strong dynamical trap effect and dotted lines match the absence of dynamical trap. Four bottom diagrams depict phase portraits and density functions of the particle in the middle of 1000-particle chain with low (label 1) and high (label 2) density of particles.

trap effect could be responsible for establishing of complex patterns of the system motion near the equilibrium point.

\section{Virtual Stick Balancing}

Inspite of these achievments until recently there were no experimental evidences of the dynamical trap effect existence in the real world. In the present paper we consider the process of balancing a virtual inverted pendulum in viscous medium. The task of human stick balancing has been investigated widely from various perspectives; studies based on both real-world and virtual experiments are available (see, e.g., $[6,7])$. However, up to now attention was mainly paid to the in-depth understanding of the mechanical and psychomotor aspects of human control, while we aim to elucidate some general properties of human cognition, considering stick balancing just as an example of a dynamical process controlled by human.

In the previous studies it was experimentally confirmed [8] that at least for certain values of the system parameters the behavior characteristics of the operator of such system are extremely similar to the patterns produced by the dynamical system described by the dynamical traps model [5]. The purpose of the current work is to generalize the obtained results by elucidating the feasible sets of the virtual experiments parameters and investigating how operator behavior characteristics vary depending on these parameters.

The motion of the analyzed virtual pendulum embedded into viscous environment with respect to its upright position is described by the following dimensionless model of the overdamped oscillator:

$$
\tau \dot{\theta}=\sin \theta-A v(t) \cos \theta .
$$

Here the phase space variable $\theta$ is the angle between the stick and the vertical axis, $\tau$ is a time scale parameter and the right-hand part of the equation represents the sum of friction and gravity force moments; $v(t)$ stands for the velocity of platform motion which is actually the control parameter affected by the system operator while $A$ is an amplifying coefficient of control effort.

It should be noted that the phase space of system (2) comprises not only angle $\theta$ but also its derivative $\dot{\theta}$. This assumption is due to the fact that the operator controlling the system evidently perceive the angular velocity of the stick and regulates the value of control effort $v(t)$ based on the current values of both factors. Hence, the system dynamics is determined not only by the stick angle but by the angular velocity as well. The similar approach of the phase space extension was previously proposed in the studies on the car following theory [4] where "position-velocity" phase space was extended by the acceleration as the third independent phase variable. In particular, a possible equation describing the 

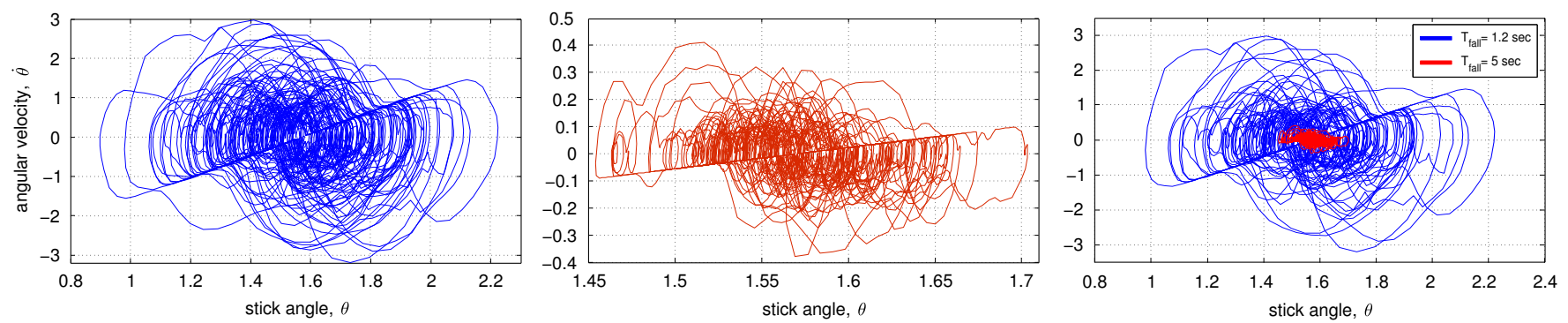

Fig. 2: Phase space trajectories of the virtual inverted pendulum motion under human control. Top frame represents the trajectory corresponding to the parameter $T_{\text {fall }}=1.2 \mathrm{sec}$, figure in the center represents trajectory for $T_{\text {fall }}=$ $5 \mathrm{sec}$, and the bottom image demonstrates the relative scales of two trajectories.

human reaction via changing the cart velocity $v$ could be of the following form

$$
\tau \dot{v}=\Omega(v)\left[V_{\text {opt }}(\theta, \dot{\theta})-v\right]+\bar{\xi}(t)
$$

where $\Omega(v)$ is some analogy to the dynamical trap factor discussed in Introduction and caused by the bounded capacity of human control over the cart velocity, $\bar{\xi}(t)$ is white noise, and the function $V_{\text {opt }}(\theta, \dot{\theta})$ describes the optimal reaction of the strictly rational operator. Leaping ahead, we may say that the found analogy between the experimental data and the data generated by the model of oscillator with dynamical traps [5] justifies the approaches based on approximations similar to Eq. (3).

In order to conduct the virtual experiments, we developed a simple software tool that implements the model described above. The operator has to maintain the angle between the virtual stick and the vertical axis near the unstable equilibrium position $\theta_{e q}=0$ by moving the platform via computer mouse.

\section{Experiments}

A few sessions of the experiments were held. During each session subjects had to control virtual inverted pendulum for the time period of 5 to 20 minutes after 5 -minutes adaptation period. To prevent the fatigue effect, sessions were held on the different days. For each participant we have acquired at least three sets of data for various durations of control process.

The numerical data captured from each subject was analyzed separately. From each raw data set obtained we extracted the data required to visualize the phase space trajectories of the inverted pendulum motion in "angle-angular velocity" phase space and variables distributions. It was discovered that for all the participants the statistical properties of the stick motion controlled by human actions have been discovered to be notably similar. Namely, the portraits formed by irregular trajectories of the virtual stick motion on the "angle-angular velocity" phase plane (Fig. 2) and corresponding phase variables distributions (Fig. 3) are of a high degree of similarity for all the operators participated in the experiments. This fact is in accordance to the previous results [8].

The main parameter of system (2) determining the behavior of the virtual pendulum is the time scale $\tau$ that may be interpreted as the value characterizing the fall time $T_{\text {fall }}$ of the stick not affected by the operator. Empirically we found the following relation between these values: $T_{\text {fall }} \approx 4 \tau$ sec. We figured out that for $T_{\text {fall }}<1 \mathrm{sec}$ all participants of the experiments failed to perform task most of the times. For $T_{\text {fall }}>4 \mathrm{sec}$ the participants demonstrated perfect performance, i.e., there were no registered failures in performing the task.

In order to figure out the dependency of the system phase portrait structure and phase variables distributions on the time scale parameter, we analyzed the data obtained during the experiments for various values of $T_{\text {fall }}$. The results of the analysis are represented on Fig. 2 and Fig. 3. It is clearly seen that for two values of $T_{\text {fall }}$ that are close to the opposite boundaries of the feasibility domain phase portraits and variables distributions of system (2) exhibit self-similar structure. For values of $T_{\text {fall }}$ in between these two values the results remain similar.

For all tested values of system parameters the structure of the phase trajectory and variables distributions produced by the virtual stick under human control are quite similar to the ones of an oscillator studied in [5] (see Fig. 1). The certain dissimilarity of the trajectories in the neighborhood of the equilibrium points is probably due to the fact that these equilibria are of different nature; the oscillators in the chain are artificially exposed to the external white noise disturbance, while the virtual stick is itself unstable at $\theta=0$.

\section{Conclusion}

The series of experiments on virtual stick balancing is presented. The feature of fuzzy rationality is shown to be present in the actions of human operator controlling both "slow" and "fast" virtual stick. The similar- 

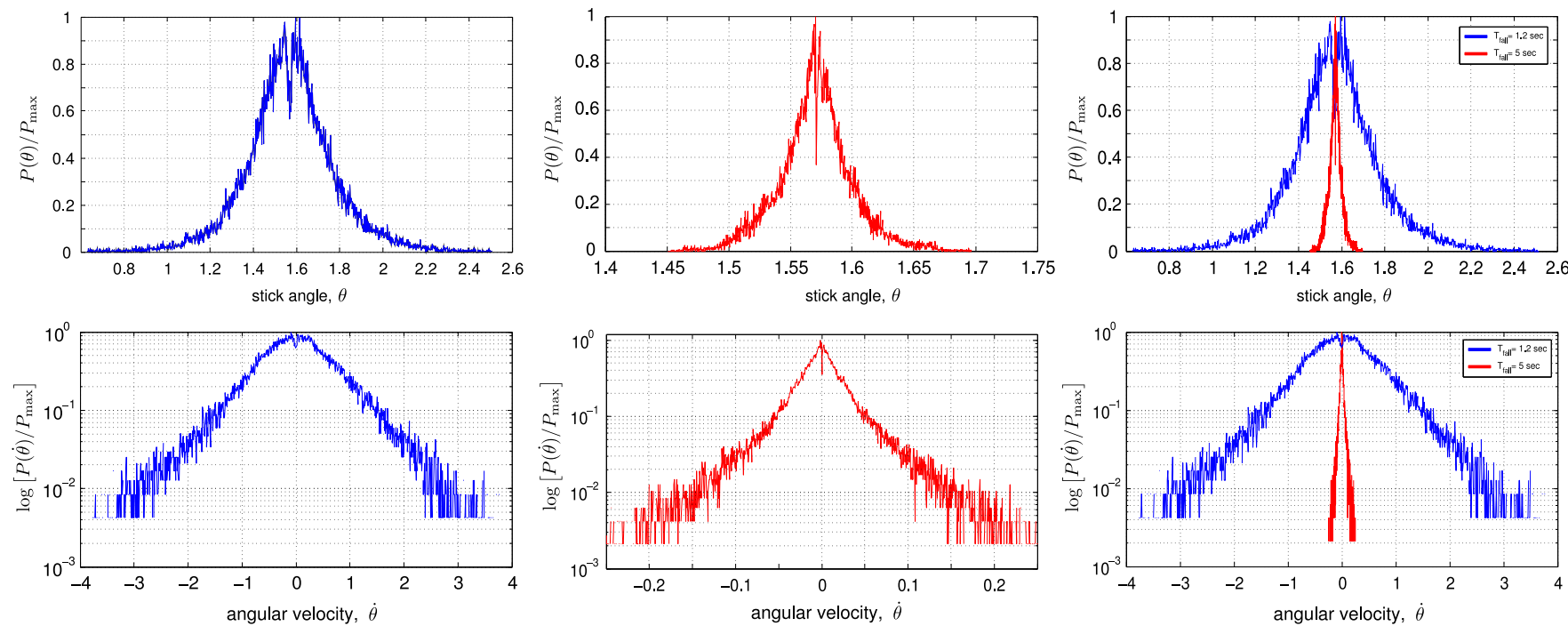

Fig. 3: Distributions of the virtual stick motion phase variables. The top three frames represent the angle distributions while the bottom three demonstrate the angular velocity distributions. In both rows the most left picture represents the distribution corresponding to the parameter $T_{\text {fall }}=1.2 \mathrm{sec}$, figure in the center represents distribution for $T_{\text {fall }}=5 \mathrm{sec}$, and the most right frame demonstrates the relative scales of two distributions

ity of the statistical characteristics of the stick motion under human control and the motion of the oscillator described by the dynamical traps model may serve as the experimental evidence of the latter model.

The obtained results enable us to state that the major properties of the given inverted pendulum dynamics controlled by human actions do not depend within scaling on the main system parameters. This fact may enable us to pose a question about the existence of the general laws describing human cognition near the reaction threshold, which must be of probabilistic nature. Moreover, one may even speculate that the results obtained give evidence to the fact that the standard notion of fixed-point attractor may not be applicable in dynamical systems where human role is crucial due to the phenomena of fuzzy rationality.

\section{Acknowledgments}

The work was supported in part by the JSPS Grants-in-Aid for Scientific Research Program, Grant 245404100001.

\section{References}

[1] R. A. Meyers (ed.) Encyclopedia of Complexity and Systems Science. SpringerScience+Business Media, LLC., New York, 2009

[2] I. Lubashevsky, Dynamical traps caused by fuzzy rationality as a new emergence mechanism, Adv. Comp. Syst., 15, p. 1250045(1-25), 2012.

[3] K. K. Dompere, Fuzzy Rationality, SpringerVerlag, Berlin, 2009.
[4] I. Lubashevsky, P. Wagner, and R. Mahnke, Bounded rational driver models, Eur. Phys. J. B, 32, pp. 243-247, 2003

[5] I. A. Lubashevsky, R. Mahnke, M. Hajimahmoodzadeh, and A. A. Katsnelson, Long-lived states of oscillator chains with dynamical traps, Eur. Phys. J. B 44, pp. 63-70, 2005.

[6] J. G. Milton, et al. Balancing with vibration: a prelude for "Drift and Act balance control. PLoS ONE 4(10), e7427, doi:10.1371/journal.pone.0007427, 2009.

[7] S. Suzuki, F. Harashima, and K. Furuta, Human control law and brain activity of voluntary motion by utilizing a balancing task with an inverted pendulum, Adv. in Hum.-Comp. Inter., p. 215825, 2010 .

[8] A. Zgonnikov, I. Lubashevsky, and M. Mozgovoy, Computer simulation of stick balancing: action point analysis. In Proceedings of the 2012 Joint International Conference on Human-Centered Computer Environments. ACM, New York, NY, USA, pp. 162-164, 2012. 